\title{
Comparison of the Melatonin Receptor Agonist Ramelteon and the Non-Benzodiazepine Hypnotic Zolpidem for Nocturia"
}

\author{
Hideki Mukouyama ${ }^{1}, K_{\text {Kimio Sugaya }}{ }^{2,3 \#}$, Saori Nishijima ${ }^{2}$, Hidekatsu Naka ${ }^{4}$, \\ Misao Sakumoto ${ }^{5}$, Tomohiro Onaga ${ }^{6}$, Katsumi Kadekawa ${ }^{2,6}$, Katsuhiro Ashitomi ${ }^{2,7}$ \\ ${ }^{1}$ Department of Urology, Nanbu Tokushukai Hospital, Okinawa, Japan \\ ${ }^{2}$ Southern Knights' Laboratory LLP, Okinawa, Japan \\ ${ }^{3}$ Kitakami Central Hospital, Okinawa, Japan \\ ${ }^{4}$ Nishizaki Hospital, Okinawa, Japan \\ ${ }^{5}$ Sakumoto Urology and Dermatology Clinic, Okinawa, Japan \\ ${ }^{6}$ Department of Urology, Okinawa Kyodo Hospital, Okinawa, Japan \\ ${ }^{7}$ Department of Urology, Okinawa Hokubu Hospital, Okinawa, Japan \\ Email: "sugaya@sklabo.com
}

Received November 4, 2013; revised November 25, 2013; accepted December 2, 2013

Copyright (C) 2013 Hideki Mukouyama et al. This is an open access article distributed under the Creative Commons Attribution License, which permits unrestricted use, distribution, and reproduction in any medium, provided the original work is properly cited. In accordance of the Creative Commons Attribution License all Copyrights @ 2013 are reserved for SCIRP and the owner of the intellectual property Hideki Mukouyama et al. All Copyright (C) 2013 are guarded by law and by SCIRP as a guardian.

\begin{abstract}
To examine the efficacy of the melatonin receptor agonist ramelteon for nocturia, it was compared with zolpidem, a conventional non-benzodiazepine hypnotic. A total of 50 patients with nocturia ( $\geq 2$ urinations/night) were enrolled. Subjects assigned odd numbers or even numbers were respectively prescribed $8 \mathrm{mg}$ of ramelteon ( $\mathrm{n}=27$; mean age: 75 years) or $5 \mathrm{mg}$ of zolpidem ( $\mathrm{n}=23$; mean age: 73 years) once a day before sleeping for 4 weeks. The daytime and nighttime frequencies of urination, as well as the results of global self-assessment by the patients, were compared between the two groups before and after 4 weeks of treatment. Both ramelteon and zolpidem caused a significant decrease of nocturia to about once per night after 4 weeks. The global self-assessment rating at 4 weeks was "good" or "fair" for more patients in the zolpidem group than in the ramelteon group, while the rating was "excellent" or "no change" for more patients in the ramelteon group. There were no serious adverse events in either group. Ramelteon was safe and effective for nocturia, achieving similar results to zolpidem. However, responders and non-responders to ramelteon were more clearly distinguished. Ramelteon might be effective for patients with sleep disturbance and nocturia because of low melatonin levels. Therefore, as diagnostic therapy for identification of nocturia caused by sleep disturbance and melatonin deficiency, ramelteon should be administered to patients who do not respond to alpha- 1 antagonists and/or anticholinergic agents.
\end{abstract}

Keywords: Ramelteon; Nocturia; Melatonin; Zolpidem

\section{Introduction}

Nocturia is common in the elderly, and is one of the most troublesome urologic symptoms [1,2]. Several prospective studies have shown that nocturia is associated with a higher mortality rate, even after adjustment for confounding factors [3]. Multiple factors may contribute to the occurrence of nocturia, including various pathologi-

${ }^{*}$ Conflict of interest: None declared.

${ }^{\#}$ Corresponding author. cal conditions such as cardiovascular disease, diabetes mellitus, lower urinary tract obstruction, anxiety disorders or primary sleep disorders, and various other behavioral and environmental factors [4-7]. Therefore, various drugs and other methods have been tried for the treatment of nocturia, including alpha-1 receptor antagonists, anticholinergic agents, nonsteroidal anti-inflammatory drugs, antidepressants (minor tranquilizers), diuretics, desmopressin, hypnotics, and melatonin [8,9]. Ramelteon was a novel hypnotic drug $[10,11]$ that was 
released recently. It is an orally active chrono hypnotic agent that acts selectively on melatonin MT1 and MT2 receptors, which are primarily located in the suprachiasmatic nucleus. In order to examine the efficacy of ramelteon $\left(\right.$ Rozerem $\left.^{\circledR}\right)$ for nocturia, we compared it with zolpidem (Myslee ${ }^{\circledR}$ ), a conventional non-benzodiazepine hypnotic (GABAa receptor modulator) [12], in elderly patients with nocturia.

\section{Materials and Methods}

A total of 50 patients with nocturia were enrolled from January 2011 to April 2012. The inclusion criterion for this study was urination $\geq 2$ times/night. Patients with a history of diseases or conditions that could affect lower urinary tract function were excluded from this study, including urinary tract surgery within 6 months, an indwelling bladder catheter, intermittent catheterization, electrical stimulation therapy, bladder training, prostatic cancer, bladder cancer, bladder stones, active urinary tract infection, and interstitial cystitis. If the patients were taking drugs for nocturia, including alpha- 1 receptor antagonists and/or anticholinergic agents, administration was continued during the study. However, patients using sedatives or hypnotics and those with a high water intake were excluded from the study. The Okinawa Kyodo Hospital Ethics Committee approved the conduct of this study on behalf of all participating facilities, and informed consent was obtained from all participants before they entered the trial.

Subjects who were assigned odd numbers or even number sat each hospital were respectively administered $8 \mathrm{mg}$ of ramelteon (Rozerem ${ }^{\circledR}$ Tablet $8 \mathrm{mg}$ ) or $5 \mathrm{mg}$ of zolpidem (Myslee ${ }^{\circledR}$ Tablet $5 \mathrm{mg}$ ) once a day before sleeping for 4 weeks. This study was not blinded. The prostate volume (in men) and the post-voiding residual urine (PVR) volume (in men and women) were measured by transabdominal ultrasonography before the start of the study. The systolic and diastolic blood pressure, heart rate, International Prostate Symptom Score (IPSS), Quality of Life (QOL) score, Overactive Bladder Symptom Score (OABSS), daytime and nighttime frequency, and PVR were evaluated before starting study and after 4 weeks of treatment. Global self-assessment ratings assigned by the patients themselves (excellent, good, fair, no change, or worse) and adverse events were also examined after 4 weeks of treatment. The overall clinical status was also compared between the ramelteon and zolpidem groups before starting study and after 4 weeks of therapy.

Results are reported as the mean \pm standard deviation (SD). Student's $t$-test and the Wilcoxon signed-ranks test were used for statistical analysis of paired or unpaired data, while differences of categorical variables were assessed with the chi-square test. In all analyses, $\mathrm{p}<0.05$ was considered to indicate statistical significance.

\section{Results}

In the ramelteon group, 27 patients were prescribed ramelteon at $8 \mathrm{mg}$ nightly, including 20 men and 7 women with a mean age of $75 \pm 8$ years (range: 58 - 94 years). Twenty-five of these 27 patients were assessed after 4 weeks of medication, while 2 patients stopped treatment because of adverse events. In the zolpidem group, 23 patients were prescribed zolpidem at $5 \mathrm{mg}$ nightly, including 14 men and 9 women with a mean age of $73 \pm 12$ years (range: 49 - 94 years). All 23 patients were assessed after 4 weeks of medication. Before starting treatment, there were no significant differences of age, systolic blood pressure, diastolic blood pressure, heart rate, complications, prostate volume, duration of nocturia, daytime and nighttime frequency, OABSS and IPSS items, total OABSS, total IPSS, and QOL score between the 2 groups (Table 1). The PVR volume was small in both groups, but was larger in the ramelteon group $(10 \pm 13$ $\mathrm{ml})$ than in the zolpidem group $(2 \pm 5 \mathrm{ml})$. Nineteen patients from the ramelteon group (70\%) and 18 patients from the zolpidem group (78\%) had already received treatment for lower urinary tract symptoms with alpha-1 antagonists, anticholinergic agents, and/or herbal medicines.

In the ramelteon group, the daytime frequency of urination decreased significantly from $8.1 \pm 3.0$ times before treatment to $7.9 \pm 3.1$ times $(n=25, p=0.045)$ after 4 weeks (Figure 1). The nighttime frequency of urination also decreased significantly from $3.8 \pm 1.4$ times before

Table 1. Demographic profile of the patients with nocturia.

\begin{tabular}{ccc}
\hline Group & Ramelteon & Zolpidem \\
\hline Case number (male:female) & $27(20: 7)$ & $23(14: 9)$ \\
Age (years) & $75 \pm 8$ & $73 \pm 12$ \\
Basal disease & & \\
Benign prostatic hyperplasia & 14 & 11 \\
Chronic prostatitis & 1 & 0 \\
Post-prostatectomy & 1 & 1 \\
Overactive bladder & 8 & 10 \\
Stress incontinence (post-op.) & 1 & 8 \\
Hypertension & 8 & 1 \\
Diabetics & 4 & 5 \\
Cerebrospinal disorders & 7 & $21 \pm \pm 20$ \\
Systolic blood pressure (mmHg) & $134 \pm 12$ & $75 \pm 9$ \\
Diastolic blood pressure (mmHg) & $74 \pm 10$ & $24 \pm 8$ \\
Heart rate (/min) & $75 \pm 9$ & $2 \pm 54$ \\
Prostate volume (ml) & $30 \pm 18$ & 10 \\
Post-voiding residual volume (ml) & $10 \pm 13$ & $41 \pm 51$ \\
\hline
\end{tabular}

Mean $\pm \mathrm{SD},{ }^{1} \mathrm{p}=0.041$. 


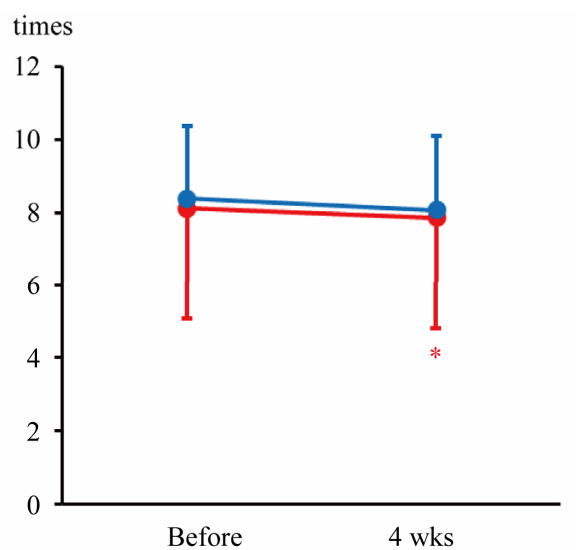

(a)

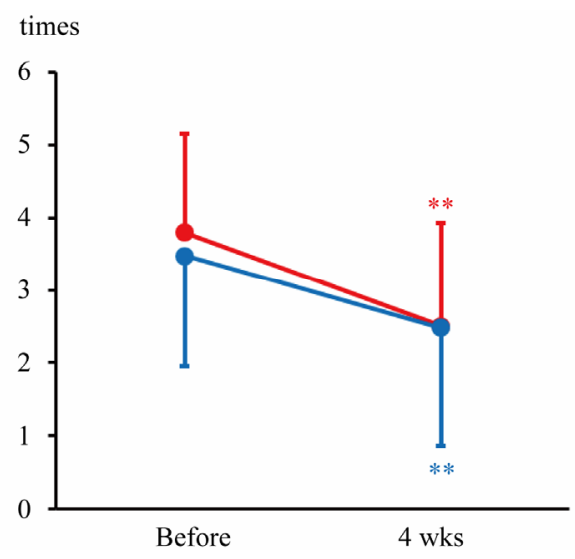

(b)

Figure 1. Frequency of urination before and after four weeks of treatment in the ramelteon and zolpidem groups. Daytime (a) and nighttime frequency (b). Red: Ramelteon, Blue: Zolpidem.

treatment to $2.5 \pm 1.4$ times $(\mathrm{n}=25, \mathrm{p}<0.001)$ after 4 weeks. With the decrease of both daytime and nighttime urination, the total OABSS and total IPSS also decreased significantly at 4 weeks (Figure 2). Furthermore, the QOL score decreased significantly from $4.7 \pm 1.0$ before treatment to $3.5 \pm 1.4$ ( $\mathrm{p}<0.001)$ after 4 weeks. The systolic blood pressure, diastolic blood pressure, heart rate, and PVR did not change. The global self-assessment rating at 4 weeks was "excellent” in 6 patients (24\%), "good" in 4 (16\%), "fair" in 5 (20\%), and "no change" in 10 (40\%) (Figure 3). None of the patients rated themselves as "worse". Adverse events were observed in 5 out of 27 patients (19\%). The symptoms noted up to 4 weeks were lassitude ( $n=3$, one dropped out at 2 weeks), unsteadiness $(n=1)$, and palpitations plus edema of lower extremities ( $n=1$, this patient dropped out at 2 weeks). These events were not serious and resolved promptly after discontinuation of ramelteon.

In the zolpidem group, the nighttime frequency of urination (but not daytime frequency) decreased significantly from $3.5 \pm 1.5$ times before treatment to $2.5 \pm 1.6$

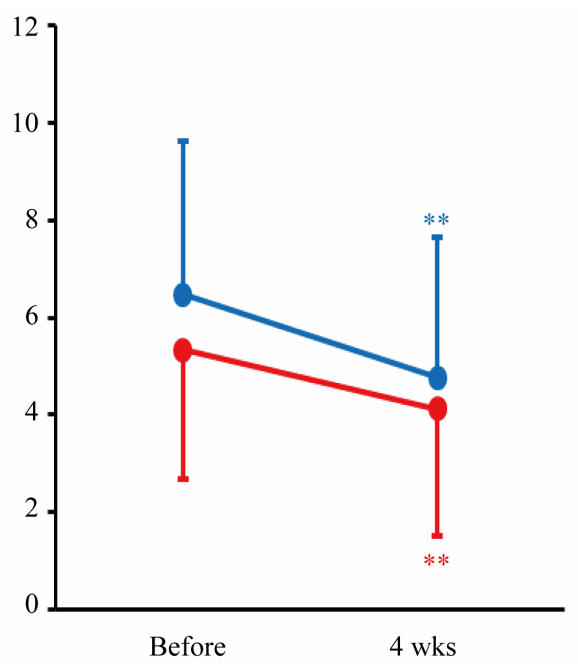

(a)

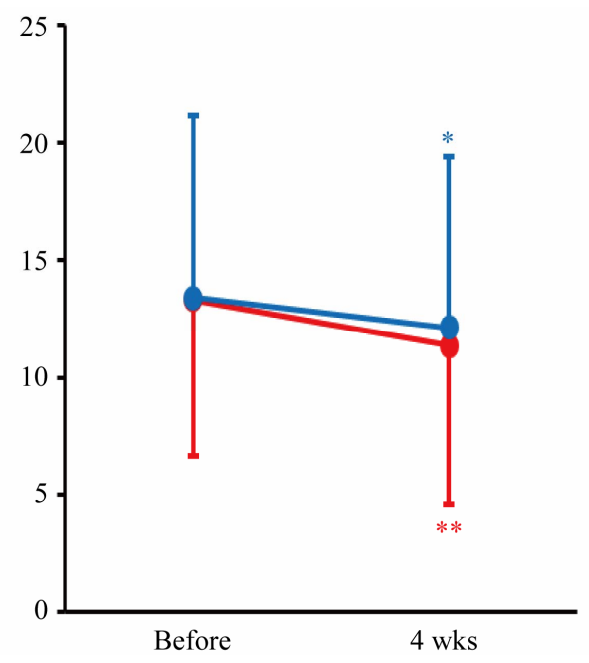

(b)

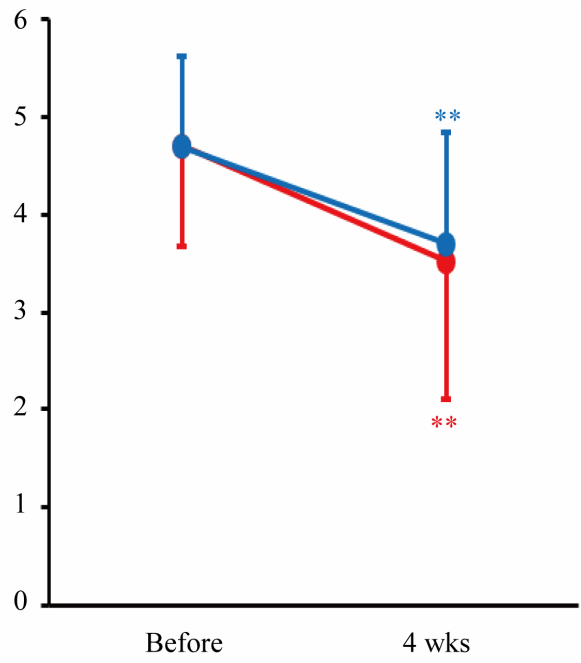

(c)

Figure 2. Total OABSS, total IPSS, and QOL score of the ramelteon and zolpidem groups. Total OABSS (a), Total IPSS (b), and QOL score (c). Red: Ramelteon, Blue: Zolpidem. 


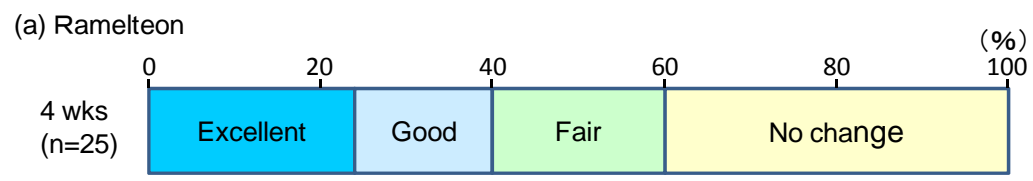

(b). Zolpidem

\begin{tabular}{|c|c|c|c|c|c|}
\hline & 20 & 40 & 60 & 80 & 100 \\
\hline $\begin{array}{l}4 \text { WKS } \\
(n=23)\end{array}$ & Good & & & No change & W \\
\hline
\end{tabular}

Figure 3. The global self-assessment ratings assigned by the patients after four weeks. Ramelteon (a) and zolpidem groups (b). W: worse.

times $(\mathrm{n}=23, \mathrm{p}<0.001)$ after 4 weeks (Figure 1). With the decrease of frequency, total OABSS and total IPSS also decreased significantly at 4 weeks (Figure 2). Furthermore, the QOL score decreased significantly from $4.7 \pm 0.9$ before treatment to $3.7 \pm 1.1(p<0.001)$ after 4 weeks. The systolic blood pressure, diastolic blood pressure, heart rate, and PVR did not change. The global selfassessment rating at 4 weeks was "good" in 6 patients (26\%), "fair" in 10 (43\%), "no change" in 6 (26\%), and "worse" in 1 (4\%) (Figure 3). No patient gave a rating of “excellent”. Adverse events were observed in 2 of the 23 patients (9\%), both of whom had lassitude at 4 weeks. These events were not serious and resolved promptly after discontinuation of zolpidem.

There were no significant differences of the daytime and nighttime frequency, total OABSS, total IPSS, QOL score, blood pressure, heart rate, PVR, and adverse events between the two groups at 4 weeks. There were also no significant between-group differences in the number of patients with "excellent" or "good" self-assessment ratings. However, there was a significantly greater difference between the number of patients with "excellent" or "no change" ratings and those with "good" or "fair" ratings in the ramelteon group compared with the zolpidem group (chi-square test, $\mathrm{p}=0.020$ ).

\section{Discussion}

In the present study, both ramelteon and zolpidem decreased nocturia to about once per night after 4 weeks of treatment. In the ramelteon group, but not the zolpidem group, the daytime frequency of urination also decreased significantly. Adverse events and drop-out were slightly more common in the ramelteon group than in the zolpidem group, but the difference was not significant and there were no serious events in either group. The number of patients with a "good" or "fair" global self-assessment rating was larger in the zolpidem group, while more patients gave a rating of "excellent" or "no change" in the ramelteon group. This difference was thought to be due to the characteristic effect of ramelteon on nocturia.

Nocturia is defined as the need to wake once or more times during the night to void [13]. Sleep disturbance is a typical cause of nocturia, and the plasma melatonin level of patients with nocturia is significantly lower than that of those without nocturia [14]. Hypnotics including zolpidem reduce the frequency of nocturia to about once nightly $[8,12,15]$, and the effect of melatonin on nocturia has been reported to be similar to that of hypnotics [8]. Many patients fear that they will be come dependent on hypnotics if they take such drugs for too long [8], so the drugs were administered for 4 weeks in this study. Ramelteon is a melatonin MT1 and MT2 receptor agonist that is recently reported to be effective for nocturia [16]. In the present study, both ramelteon and zolpidem proved nocturia in patients who had shown a poor response to alpha-1 antagonists, anticholinergic agents, and/or herbal medicines.

In the ramelteon group, fewer patients gave a global self-assessment rating of "good" or "fair" compared with ratings of "excellent" or "no change”. Although there was no significant difference, the frequency of adverse events and drop-out was higher in the ramelteon group than in the zolpidem group. Therefore, there was a clear difference between patients who responded or failed to respond to ramelteon. When various morning parameters were compared between subjects who were troubled by nocturnal urination (nocturia) and those who were not, the plasma melatonin level was significantly lower in the former group [17], suggesting that persons with nocturia are likely to have sleep disturbance. The present results may indicate that ramelteon is effective for patients with sleep disturbance and nocturia due to low melatonin levels. However, routine measurement of the plasma melatonin level in patients with nocturia is not feasible. Therefore, ramelteon could be administered to patients in whom nocturia is not improved by alpha-1 antagonists and/or anticholinergic agents as diagnostic therapy for nocturia associated with sleep disturbance and low melatonin levels.

In the ramelteon group, but not the zolpidem group, the daytime frequency of urination decreased significantly. When $2 \mathrm{mg}$ of melatonin was administered to patients with nocturia before sleeping, the morning plasma melatonin level was very high [8] compared with that 
in persons without nocturia [14]. Melatonin inhibits the contraction of isolated rat bladder smooth muscle [18], and increases bladder capacity in rats [19]. Therefore, administering melatonin or ramelteon before sleeping might also have a beneficial influence on lower urinary tract function in the daytime.

\section{Conclusion}

Ramelteon was safe and effective for nocturia, achieving similar results to zolpidem, although responders and non-responders to ramelteon were more clearly distinguished. Ramelteon may be effective for patients who have sleep disturbance and nocturia associated with low melatonin levels. Accordingly, ramelteon could be used as diagnostic therapy for nocturia caused by sleep disturbance and low melatonin levels in patients whose nocturia is not improved by alpha-1 antagonists and/or anticholinergic agents.

\section{REFERENCES}

[1] M. H. Blanker, A. M. Bohnen, F. P. Groeneveld, R. M. Bernsen, A. Prins and J. L. Ruud Bosch, "Normal Voiding Patterns and Determinants of Increased Diurnal and Nocturnal Voiding Frequency in Elderly Men,” Journal of Urology, Vol. 164, No. 4, 2000, pp. 1201-1205. http://dx.doi.org/10.1016/S0022-5347(05)67141-8

[2] M. H. Blanker, R. M. Bernsen, J. L. Bosch, S. Thomas, F. P. Groeneveld, A. D. Prins and A. M. Bohnen, "Relation between Nocturnal Voiding Frequency and Nocturnal Urine Production in Older Men,” Urology, Vol. 60, No. 4, 2002, pp. 612-616.

http://dx.doi.org/10.1016/S0090-4295(02)01818-6

[3] K. Yoshimura, "Correlates for Nocturia: A Review of Epidemiological Studies,” Journal of Urology, Vol. 19, No. 4, 2012, pp. 317-329.

[4] J. P. Weiss, J. G. Blaivas, D. S. Stember and M. M. Brooks, "Nocturia in Adults. Etiology and Classification," Neurourology and Urodynamics, Vol. 17, No. 5, 1998, pp. 467472.

http://dx.doi.org/10.1002/(SICI)1520-6777(1998)17:5<46 7::AID-NAU2>3.0.CO;2-B

[5] J. P. Weiss, J. G. Blaivas, D. S. Stember and D. C. Chaikin, "Evaluation of the Etiology of Nocturia in Men: The Nocturia and Nocturnal Bladder Capacity Indices," Neurourology and Urodynamics, Vol. 18, No. 6, 1999, pp. 559-565.

http://dx.doi.org/10.1002/(SICI)1520-6777(1999)18:6<55 9::AID-NAU6>3.0.CO;2-D

[6] K. Yoshimura, N. Terada, Y. Matsui, A. Terai, N. Kinukawa and Y. Arai, "Prevalence of and Risk Factors for Nocturia: Analysis of a Health Screening Program," International Journal of Urology, Vol. 11, No. 5, 2004, pp. 282-287.

http://dx.doi.org/10.1111/j.1442-2042.2004.00791.x

[7] L. W. Gourova, C. van de Beek, M. G. Spigt, F. H. Nieman and P. E. van Kerrebroeck, "Predictive Factors for Nocturia in Elderly Men: A Cross-Sectional Study in 21 General Practices,” BJU International, Vol. 97, No. 3, 2006, pp. 528-532. http://dx.doi.org/10.1111/j.1464-410X.2006.06029.x

[8] K. Sugaya S. Nishijima, M. Miyazato, K. Kadekawa and Y. Ogawa, "Effects of Melatonin and Rilmazafone on Nocturia in the Elderly,” Journal of International Medical Research, Vol. 35, No. 5, 2007, pp. 685-691.

[9] O. Nishizawa, I. Araki, O. Ishizuka, N. Uchimura, H. Oh-oka, S. Ozono, H. Kakizaki, M. Kasahara, M. Gotoh, R. Sakakibara, H. Shinbo, K. Sugaya, Y. Suzuki, A. Sone, M. Takei, M. Takeda, A. Hirayama, Y. Homma, O. Yamaguchi, T. Yamanishi, O. Yokoyama and M. Yoshida, “Clinical Guidelines for Nocturia,” International Journal of Urology, Vol. 17, No. 5, 2010, pp. 379-409.

[10] G. Zammit, S. Wang-Weigand, M. Rosenthal and X. Peng, "Effect of Ramelteon on Middle-of-the-Night Balance in Older Adults with Chronic Insomnia,” Journal of Clinical Sleep Medicine, Vol. 15, No. 1, 2009, pp. 34-40.

[11] S. R. Pandi-Perumal, V. Srinivasan, D. W. Spence, A. Moscovitch, R. Hardeland, G. M. Brown and D. P. Cardinali, "Ramelteon: A Review of Its Therapeutic Potential in Sleep Disorders,” Advances in Therapy, Vol. 26, No. 6, 2009, pp. 613-626. http://dx.doi.org/10.1007/s12325-009-0041-6

[12] Y. S. Song and J. H. Ku, “Zolpidem Pharmacotherapy Combined with Alpha-Blocker Therapy for Nocturia Unresponsive to Alpha-Blocker Monotherapy in Men with Lower Urinary Tract Symptoms: A Preliminary Study," International Urology and Nephrology, Vol. 39, No. 4, 2007, pp. 1147-1152.

[13] P. Abrams, L. Cardozo, M. Fall, D. Griffiths, P. Rosier, U. Ulmsten, P. Van Kerrebroeck, A. Victor and A. Wein, "Standardisation Sub-Committee of the International Continence Society: The Standardisation of Terminology in Lower Urinary Tract Function: Report from the Standardisation Sub-Committee of the International Continence Society," Urology, Vol. 61, No. 1, 2003, pp. 37-49. http://dx.doi.org/10.1016/S0090-4295(02)02243-4

[14] K. Sugaya, S. Nishijima, M. Oda, T. Owan, M. Miyazato and Y. Ogawa, "Biochemical and Body Composition Analysis of Nocturia in the Elderly," Neurourology and Urodynamics, Vol. 27, No. 3, 2008, pp. 205-211. http://dx.doi.org/10.1002/nau.20492

[15] K. Fujikawa, M. Kasahara, Y. Matsui and H. Takeuchi, "Human Atrial Natriuretic Peptide Is a Useful Criterion in Treatment of Nocturia," Scandinavian Journal of Urology and Nephrology, Vol. 35, No. 4, 2001, pp. 310-313. http://dx.doi.org/10.1080/003655901750425909

[16] N. Shimizu, M. Nozawa, Y. Itami, Y. Yamamoto and Y. Hayashi, "A Study on Efficacy of Ramelteon in Patients with Insomnia and Nocturia,” Japanese Journal of Urological Surgery, Vol. 24, 2011, pp. 1473-1479.

[17] K. Sugaya, S. Nishijima, M. Miyazato, T. Owan, Y. Oshiro, A. Uchida, S. Hokama and Y. Ogawa, "Investigation of Biochemical Factors Related to Non-Bothersome Nocturnal Urination,” BioMed Research, Vol. 28, No. 4, 2007, pp. 458-460. http://dx.doi.org/10.2220/biomedres.28.213

[18] J. H. Han, I. H. Chang, S. C. Myung, M. Y. Lee, W. Y. 
Kim, S. Y. Lee, S. W. Lee and K. D. Kim, “A Novel Pathway Underlying the Inhibitory Effects of Melatonin on Isolated Rat Urinary Bladder Contraction,” The Korean Journal of Physiology \& Pharmacology, Vol. 16, No. 1, 2012, pp. 37-42.

http://dx.doi.org/10.4196/kjpp.2012.16.1.37
[19] Y. Matsuta, A. Yusup, K. Tanase, H. Ishida, H. Akino and O. Yokoyama, "Melatonin Increases Bladder Capacity via GABAergic System and Decreases Urine Volume in Rats,” Journal of Urology, Vol. 184, No. 1, 2010, pp. 386-391. http://dx.doi.org/10.1016/j.juro.2010.03.002 